\title{
Collisional semiclassical approximations in phase-space representation
}

\author{
G. W. Bund, ${ }^{1, *}$ S. S. Mizrahi, ${ }^{2, \dagger}$ and M. C. Tijero ${ }^{1,3, \$}$ \\ ${ }^{1}$ Instituto de Física Teórica, Universidade Estadual Paulista, Rua Pamplona 145, 01405-900 São Paulo, São Paulo, Brazil \\ ${ }^{2}$ Departamento de Física, Universidade Federal de São Carlos, Rodovia Washington Luiz Km 235, 13565-905 São Carlos, \\ São Paulo, Brazil \\ ${ }^{3}$ Pontifícia Universidade Católica (PUC), São Paulo, São Paulo, Brazil
}

(Received 31 January 1997)

\begin{abstract}
The Gaussian wave-packet phase-space representation is used to show that the expansion in powers of $\hbar$ of the quantum Liouville propagator leads, in the zeroth-order term, to results close to those obtained in the statistical quasiclassical method of Lee and Scully in the Weyl-Wigner picture. It is also verified that, propagating the Wigner distribution along the classical trajectories, the amount of error is less than that coming from propagating the Gaussian distribution along classical trajectories. [S1050-2947(97)08309-1]

PACS number(s): $34.10 .+x, 03.65 . S q$
\end{abstract}

\section{INTRODUCTION}

Nowadays, the advantages and difficulties for using the phase-space formulation of quantum mechanics are well known [1-3]. This formulation remains still very useful for studying the classical limit of quantum mechanics as well as for describing semiclassical approximations in collisional processes; for these purposes many authors use the WeylWigner (WW) picture [1,4-6]. For the collisional problem, it is often combined with the quasiclassical method of Ref. [7]. Following this approach, Lee and Scully [1] improved the accuracy of this method successfully with their statistical quasiclassical (SQC) method which was first suggested by Heller [8]. As we showed in a previous paper [9], the approach of Lee and Scully corresponds to the zeroth-order term of the expansion of the quantum Liouvillian in powers of $\hbar$ in the WW picture. So their calculated transition probabilities could have higher-order corrections.

The aim of this paper is to show that the use of the Gaussian wave-packet (GWP) phase-space representation [3,10,11] gives for the zeroth-order term, which corresponds to what we call the causal approximation (CA), results similar to, although not as good as, those obtained in the WW representation [1].

In the derivation of the expansion of the quantum Liouvillian in the GWP picture all orders of $\hbar$ are included; the first-order term we shall call the quasicausal approximation (QCA). As is shown in Refs. [10,11], one of the features of the GWP representation is that the quantum fluctuations of the mapped physical quantities become more evident. Also, the covariant distribution function for a pure state, which is one of the mappings in this representation, is usually a smooth function and always non-negative, whereas the contravariant mapping is singular, being actually an ultradistribution (see Appendix B).

Here we are also interested in verifying numerically, for the collisional problem, the statement put forward by Lee

\footnotetext{
*Electronic address: bund@axp.ift.unesp.br

†Electronic address: salomon@ power.ufscar.br

FElectronic address: maria@axp.ift.unesp.br
}

[3]: "the amount of error arising from propagating the Wigner distribution function (WDF) along the classical trajectories is usually considerably less than that coming from propagating other distributions along classical trajectories." In our case the other distribution is the GWP distribution [10-12]. It is in agreement with Lee's statement the result which we derive in Sec. II: first-order corrections in $\hbar$ in the SQC method do not improve the numerical results of Lee and Scully [1].

In our comparison of the Weyl-Wigner formalism with the GWP approach we are going to work with a collinear nonreactive collision of an atom with a diatomic molecule, the interaction between them being an exponential repulsion, first used by Secrest and Johnson [13], of the form $V=V_{0} \exp [-\alpha(x-y)]$, where the constant $V_{0}$ is related to the classical turning point of the trajectory of the particle but its value does not have any effect on the results. In the WW and GWP formalisms we have taken this potential, the definition of the $x$ and $y$ coordinates, as well as the value of $\alpha$ and $V_{0}$ from Ref. [13]; the parameter $\alpha$ is adjusted by published experimental data [13]. The mapped Hamiltonian in the GWP formalism has the constant $V_{0}$ renormalized.

In Sec. II we summarize our previous paper (Ref. [9]). In Sec. III we introduce the GWP representation and the CA and QCA. In Sec. IV we derive expressions for the transition probabilities and numerical results, and conclusions are presented in Sec. V. The appendixes contain details of expressions used in the text.

\section{CAUSAL AND QUASICAUSAL APPROXIMATIONS IN THE WW FORMALISM}

In the first part of this section we give a review of Ref. [9]. The quantum Liouville equation in the WW picture is [14]

$$
\frac{\partial W(q, p, t)}{\partial t}=-i \mathcal{L}_{Q} W(q, p, t)
$$

where $(q, p)$ is a point of phase space, and $W(q, p, t)$ is the Wigner distribution function (WDF). We use just one dimension. The quantum Liouvillian is 


$$
\mathcal{L}_{Q}=H(q, p)\left[i \frac{2}{\hbar} \sin \frac{\hbar}{2} \overleftrightarrow{\Lambda}\right],
$$

$H(q, p)$ being the Hamiltonian of the system, and the operator

$$
\overleftrightarrow{\Lambda}=\frac{\grave{\partial}}{\partial q} \frac{\vec{\partial}}{\partial p}-\frac{\grave{\partial}}{\partial p} \frac{\vec{\partial}}{\partial q}
$$

is the Poisson bracket; arrows indicate on which side the derivatives operate.

The formal solution of Eq. (1) is given by

$$
W(q, p, t)=e^{-i \mathcal{L}_{Q}{ }^{\left(t-t_{0}\right)}} W_{0}(q, p),
$$

where $W_{0}(q, p)$ is the WDF at the initial time $t_{0}$. Taking the classical limit of $\mathcal{L}_{Q}$ in Eq. (2), we get

$$
\mathcal{L}_{\mathrm{cl}}=i H(q, p) \stackrel{\leftrightarrow}{\Lambda},
$$

and Eq. (4) becomes [9]

$W^{(0)}(q, p, t)=e^{-i \mathcal{L}_{\mathrm{cl}}\left(t-t_{0}\right)} W_{0}(q, p)=W_{0}\left[q\left(t_{0}-t\right), p\left(t_{0}-t\right)\right]$.

Thus each point $(q, p)$ of the phase space of the initial WDF evolves classically according to Hamilton's equations, following a classical trajectory reversed in time. This we call the causal approximation.

Still, according to Ref. [9], we can make an expansion of $\mathcal{L}_{Q}$ in a power series of $\hbar^{2}$ which is substituted into Eq. (1), giving

$$
\frac{\partial W(q, p, t)}{\partial t}+i \mathcal{L}_{0} W(q, p, t)=-i \sum_{n=1}^{\infty} \hbar^{2 n} \mathcal{L}_{2 n} W(q, p, t),
$$

where $\mathcal{L}_{0}=\mathcal{L}_{\mathrm{cl}}$ and

$$
\mathcal{L}_{2 n}=H(q, p)\left[i \frac{(-1)^{n}}{2^{2 n}(2 n+1) !}(\overleftrightarrow{\Lambda})^{2 n+1}\right] .
$$

The integral equation corresponding to Eq. (7) is given by

$$
\begin{aligned}
W(q, p, t)= & e^{-i \mathcal{L}_{0}\left(t-t_{0}\right)} W_{0}(q, p) \\
& -i \sum_{n=1}^{\infty} \hbar^{2 n} \int_{t_{0}}^{t} d t^{\prime} e^{-i \mathcal{L}_{0}\left(t-t^{\prime}\right)} \mathcal{L}_{2 n} W\left(q, p, t^{\prime}\right) .
\end{aligned}
$$

Solving this equation iteratively, in first order we obtain the quasicausal approximation

$$
\begin{aligned}
W_{\mathrm{QCA}}(q, p, t)= & e^{-i \mathcal{L}_{0}\left(t-t_{0}\right)} W_{0}(q, p)-i \hbar^{2} \int_{t_{0}}^{t} d t^{\prime} e^{-i \mathcal{L}_{0}\left(t-t^{\prime}\right)} \\
& \times \mathcal{L}_{2} e^{-i \mathcal{L}_{0}\left(t^{\prime}-t_{0}\right)} W_{0}(q, p)
\end{aligned}
$$

In Eq. (10) the operator $\exp \left[-i \mathcal{L}_{0}\left(t-t_{0}\right)\right]$ is responsible for the classical character of the evolution between different times.
This formalism is applied in Ref. [9] to a collisional process where a molecule suffers a collision from a pointlike projectile. As a result, the molecule is transferred from the initial discrete energy level $|i\rangle$ to the final level $|f\rangle$; the total probability for this transition, in the limit $t \rightarrow \infty$ and $t_{0} \rightarrow-\infty$, is given by

$$
\begin{aligned}
\mathcal{P}_{i \rightarrow f}= & 2 \pi \hbar \int_{-\infty}^{\infty} d p_{0} \int_{-\infty}^{\infty} d q_{0} W_{i}\left(q_{0}, p_{0}\right) e^{i \mathcal{L}_{\left.Q^{\left(t-t_{0}\right.}\right)}} \\
& \times W_{f}\left(q_{0}, p_{0}\right) .
\end{aligned}
$$

Equation (11) is an exact result. Now introducing the QCA, in the limit $t \rightarrow \infty$ and $t_{0} \rightarrow-\infty$ we obtain

$$
\begin{aligned}
\mathcal{P}_{i \rightarrow f}^{\mathrm{QCA}}= & 2 \pi \hbar\left[\int_{-\infty}^{\infty} d p_{0} \int_{-\infty}^{\infty} d q_{0} W_{i}\left(q_{0}, p_{0}\right)\right. \\
& \times W_{f}\left[q\left(t-t_{0}\right), p\left(t-t_{0}\right)\right]+i \hbar^{2} \int_{-\infty}^{\infty} d p_{0} \\
& \times \int_{-\infty}^{\infty} d q_{0} W_{i}\left(q_{0}, p_{0}\right) \int_{t_{0}}^{t} d t^{\prime} e^{i \mathcal{L}_{0}\left(t-t^{\prime}\right)} \\
& \left.\times \mathcal{L}_{2} W_{f}\left[q\left(t^{\prime}-t_{0}\right), p\left(t^{\prime}-t_{0}\right)\right]\right],
\end{aligned}
$$

where the first term corresponds to the $\mathrm{CA}$, and the second one to the QCA. The CA corresponds to the SQC method of Lee and Scully given in Ref. [1]. Here, as in Ref. [1], the $\mathrm{H}_{2}-\mathrm{He}$ collision is considered, $\mathrm{H}_{2}$ and $\mathrm{He}$ being treated as an harmonic oscillator and a free particle, respectively. The Hamiltonian in the Weyl-Wigner phase space is given by

$$
H(Q, q, P, p)=\frac{P^{2}}{2 M}+\frac{p^{2}}{2 m}+\frac{1}{2} k q^{2}+V_{0} e^{-\alpha(Q-q)},
$$

where $Q$ and $q$ are the translational and vibrational coordinates respectively, $P$ and $p$ being their respective momenta. All the parameters appearing in Eq. (13), $M, m, V_{0}, \alpha$, and $k=m \omega^{2}$ (elastic constant of the oscillator), are taken from Ref. [13].

Following Lee and Scully (Refs. [1,3]) $Q, q, P$, and $p$ obey Hamilton's equations, so they describe classical trajectories; the initial state of the system in Eq. (12) is given by the WDF $W_{i}\left(q_{0}, p_{0}\right)$ for the harmonic oscillator; and the pair $\left(q_{0}, p_{0}\right)$ refers to the initial position and momentum of the harmonic oscillator, and it belongs to a two-dimensional rectangular grid whose size and density depend on the desired accuracy.

Integrating numerically Hamilton's equations for each $\left(q_{0}, p_{0}\right)$ of the grid for the harmonic oscillator and the appropriate initial $Q$ and $P$ of the particle, we obtain the set of final pairs $(q, p)$ for the oscillator and final $(Q, P)$ for the particle, initial and final $Q$ must be taken sufficiently large so that the particle can be considered free, which can be verified by using the fact that the total energy must be conserved along the trajectories.

In Eq. (12) the final state of the system is now given by the $\operatorname{WDF} W_{f}(q, p)$ calculated for all final phase-space points 
of the grid. Once initial and final WDF's are calculated for each point, the CA can be obtained using the first term in Eq. (12).

In order to obtain the QCA, the second term in Eq. (12) must be calculated. This term can be approximated by [12]

$$
C_{\mathrm{QCA}}=B F_{i f} \int_{t_{0}}^{t} d t^{\prime} e^{-\alpha\left[Q\left(t^{\prime}-t_{0}\right)-q\left(t^{\prime}-t_{0}\right)\right]},
$$

with the constant $B=\pi(\hbar \alpha)^{3} V_{0} / 12$, and

$$
F_{i f}=\int_{-\infty}^{\infty} d p_{0} \int_{-\infty}^{\infty} d q_{0} W_{i}\left(q_{0}, p_{0}\right) e^{i\left(t-t_{0}\right) \mathcal{L}_{0}} \frac{\partial^{3}}{\partial p_{0}^{3}} W_{f}\left(q_{0}, p_{0}\right) .
$$

Making $y=2 E_{\mathrm{OH}} / \hbar \omega\left[E_{\mathrm{OH}}\left(q_{0}, p_{0}\right)\right.$ being the energy of the classical harmonic oscillator], we have

$$
\frac{\partial^{3}}{\partial p_{0}^{3}} W_{f}\left(q_{0}, p_{0}\right)=A_{0} p_{0}^{3}+B_{0} p_{0}
$$

where

$$
A_{0}=\left(\frac{2}{\hbar \omega m}\right)^{3} \frac{\partial^{3} w_{f}(y)}{\partial y^{3}} \text { and } B_{0}=3\left(\frac{2}{\hbar \omega m}\right)^{2} \frac{\partial^{2} w_{f}(y)}{\partial y^{2}},
$$

being $w_{f}(y)=W_{f}\left(q_{0}, p_{0}\right)$.

Now we are going to show that this correction to the CA, given by Eq. (14), oscillates periodically in time $t$. Let $t_{M}$ be the time at which the atom is considered to be a free particle after colliding with the molecule. At this time the coordinate and momentum of the classical harmonic oscillator are given by

$$
\begin{aligned}
q_{0 M}= & A \cos \left(\omega t_{M}+\phi_{0}\right) \quad \text { and } \\
& p_{0 M}=-m \omega A \sin \left(\omega t_{M}+\phi_{0}\right) .
\end{aligned}
$$

At a later time $t=t_{M}+\Delta t$, with $\Delta t>0$, the momentum $p_{0}(t)$ of the harmonic oscillator will be

$$
p_{0}(t)=p_{0 M} \cos (\omega \Delta t)-m \omega q_{0 M} \sin (\omega \Delta t) .
$$

Thus, making $a=p_{0 M}$ and $b=-m \omega q_{0 M}$, one has

$$
\begin{aligned}
p_{0}^{3}(t)= & a^{3} \cos ^{3} \omega \Delta t+b^{3} \sin ^{3} \omega \Delta t \\
& +3 a b^{2} \cos \omega \Delta t \sin ^{2} \omega \Delta t \\
& +3 a^{2} b \sin \omega \Delta t \cos ^{2} \omega \Delta t .
\end{aligned}
$$

From Eqs. (17) and (18) we obtain, for Eq. (16),

$$
\begin{aligned}
A_{0} p_{0}^{3}(t)+B_{0} p_{0}(t)= & a_{3} \cos 3 \omega \Delta t+b_{3} \sin 3 \omega \Delta t \\
& +a_{1} \cos \omega \Delta t+b_{1} \sin \omega \Delta t,
\end{aligned}
$$

where

$$
\begin{gathered}
a_{3}=A_{0}\left(\alpha^{3}+\alpha^{* 3}\right), \quad b_{3}=i A_{0}\left(\alpha^{3}-\alpha^{* 3}\right), \\
a_{1}=\left(3 A_{0} \alpha \alpha^{*}+B_{0}\right)\left(\alpha+\alpha^{*}\right), \\
b_{1}=i\left(3 A_{0} \alpha \alpha^{*}+B_{0}\right)\left(\alpha-\alpha^{*}\right),
\end{gathered}
$$

and $\alpha=\frac{1}{2}(a-i b)$. Equation (19) is a Fourier's series, which substituted into Eq. (15) shows that $C_{\mathrm{QCA}}$ given by Eq. (14) is a periodical function, since in this equation the integral in the time converges in the limit $t \rightarrow \infty$. The average of this periodical function over one period of the oscillator will be zero. In this derivation we have used the approximate expression for the correction of the transition rate given by Eq. (14). The exact demonstration, although more involved, follows along similar lines.

\section{CAUSAL AND QUASICAUSAL APPROXIMATIONS IN THE GWP FORMALISM}

In the GWP representation [10-12], operators can be mapped both into covariant (CV) and contravariant (CTV) forms, and there are expressions which relate the $\mathrm{CV}$ with the CTV forms as well as both of them with the corresponding WW representation.

The commutator of two operators of the Hilbert space $A$ and $B$ in the $\mathrm{CV}$ form is written $[3,10,11]$

$$
\langle p q|[A, B]| p q\rangle=\mathcal{A}^{\mathrm{CV}}(q, p)\left(\stackrel{\leftrightarrow}{\Gamma}-\stackrel{\leftrightarrow}{\Gamma}^{*}\right) \mathcal{B}^{\mathrm{CV}}(q, p),
$$

where $(q, p)$ is a point in a phase space, $|p q\rangle$ represents the minimum uncertainty Gaussian wave-packet or coherent state, and $\mathcal{A}^{\mathrm{CV}}(q, p)$ and $\mathcal{B}^{\mathrm{CV}}(q, p)$ are the $\mathrm{CV}$ forms of operators $A$ and $B$ and $\stackrel{\leftrightarrow}{\Gamma}=\exp \left[(\hbar / 2) \stackrel{\leftarrow}{D} \vec{D}^{*}\right]$, where $D=\left(1 / a_{0}\right) \partial / \partial q-i a_{0} \partial / \partial p$. Arrows indicate on which side operators act and $a_{0}$ is a constant with dimensions $M^{1 / 2} T^{-1 / 2}$

The quantum Liouville equation in the $\mathrm{CV}$ representation for the density operator $P^{\mathrm{CV}}(q, p, t)=\langle p q \mid \Psi(t)\rangle\langle\Psi(t) \mid p q\rangle$ is given by

$$
\frac{\partial}{\partial t} P^{\mathrm{CV}}(q, p, t)=-i \mathcal{L}^{\mathrm{CV}} P^{\mathrm{CV}}(q, p, t)
$$

$\mathcal{L}^{\mathrm{CV}}$ being the quantum Liouvillian in the $\mathrm{CV}$ form,

$$
\mathcal{L}^{\mathrm{CV}}=\frac{1}{\hbar} \mathcal{H}^{\mathrm{CV}}(q, p)\left(\stackrel{\leftrightarrow}{\Gamma}-\stackrel{\leftrightarrow}{\Gamma}^{*}\right)
$$

and $\mathcal{H}^{\mathrm{CV}}(q, p)=\langle p q|H| p q\rangle$ the $\mathrm{CV}$ form of the Hamiltonian $H[11]$.

Now expanding $\mathcal{L}^{\mathrm{CV}}$ in an $\hbar$ power series, $\mathcal{L}^{\mathrm{CV}}$ $=\sum_{n=0}^{\infty} \hbar^{n} \mathcal{L}_{n}$, we identify

$$
\mathcal{L}_{n}=\frac{i}{2^{n}(n+1) !} \operatorname{Im}\left[\left(D^{n+1} \mathcal{H}^{\mathrm{CV}}\right)\left(\vec{D}^{*}\right)^{n+1}\right],
$$

where $\mathcal{L}_{0}=\mathcal{L}_{c l}$ is the classical Liouvillian.

One defines a Green's function [11] by

$$
w\left(q, p, t \mid q_{0}, p_{0}, t_{0}\right)=e^{-i\left(t-t_{0}\right) \mathcal{L}^{\mathrm{CV}}} w\left(q, p, t_{0} \mid q_{0}, p_{0}, t_{0}\right)
$$

with the condition

$$
\lim _{t \rightarrow t_{0}} w\left(q, p, t \mid q_{0}, p_{0}, t_{0}\right)=\delta\left(q-q_{0}\right) \delta\left(p-p_{0}\right) .
$$




$$
\frac{\partial}{\partial t} w\left(q, p, t \mid q_{0}, p_{0}, t_{0}\right)=-i \mathcal{L}^{\mathrm{CV}} w\left(q, p, t \mid q_{0}, p_{0}, t_{0}\right), \quad t>t_{0}
$$

where the pairs $\left(q_{0}, p_{0}\right)$ and $(q, p)$ are the momenta and the coordinates at times $t_{0}$ and $t$, respectively. Here, as discussed in Ref. [9], classical causality is broken in the $\hbar$ power-series expansion of $\mathcal{L}^{\mathrm{CV}}$ when terms with $n \geqslant 1$ are retained.

Because of Eq. (23), Eq. (26) can be written as follows:

$$
\begin{aligned}
\frac{\partial}{\partial t} w\left(t \mid t_{0}\right)+i \mathcal{L}_{0} w\left(t \mid t_{0}\right)= & \frac{2}{\hbar} \sum_{n=1}^{\infty} \frac{\left(\frac{\hbar}{2}\right)^{n+1}}{(n+1) !} \operatorname{Im}\left[\left(D^{n+1} \mathcal{H}^{\mathrm{CV}}\right)\right. \\
& \left.\times\left(D^{*}\right)^{n+1} w\left(t \mid t_{0}\right)\right]
\end{aligned}
$$

In the GWP phase space the CV form of the density operator $P^{\mathrm{CV}}(q, p, t)$ is given by $[11]$

$$
\begin{aligned}
P^{\mathrm{CV}}(q, p, t)= & \int_{-\infty}^{\infty} d p_{0} \int_{-\infty}^{\infty} d q_{0} w\left(q, p, t \mid q_{0}, p_{0}, t_{0}\right) \\
& \times P^{\mathrm{CV}}\left(q_{0}, p_{0}, t_{0}\right),
\end{aligned}
$$

so, if a formal solution of Eq. (27),

$$
\begin{aligned}
w\left(t \mid t_{0}\right)= & e^{-i\left(t-t_{0}\right) \mathcal{L}_{0}} w\left(t_{0} \mid t_{0}\right)+\frac{2}{\hbar} \int_{t_{0}}^{t} d t^{\prime} e^{-i\left(t-t^{\prime}\right) \mathcal{L}_{0}} \\
& \times \sum_{n=1}^{\infty} \frac{\left(\frac{\hbar}{2}\right)^{n+1}}{(n+1) !} \operatorname{Im}\left[\left(D^{n+1} \mathcal{H}^{\mathrm{CV}}\right)\right. \\
& \left.\times\left(D^{*}\right)^{n+1} w\left(t^{\prime} \mid t_{0}\right)\right]
\end{aligned}
$$

is substituted into Eq. (28), keeping in mind that $w\left(t_{0} \mid t_{0}\right)=\delta\left(q-q_{0}\right) \delta\left(p-p_{0}\right)$, the first term gives, for $P^{\mathrm{CV}}(q, p, t)$, the CA, while by including the lowest correction, the $n=1$ term, the QCA is obtained.

\section{TRANSITION PROBABILITIES FOR A COLLISIONAL PROCESS}

When we have a system in a given initial state $|i\rangle$, at time $t_{0}$ and final state $|f\rangle$ at time $t$, with $t>t_{0}$, the transition probability $\mathcal{P}_{i \rightarrow f}$ may be written [11]

$$
\begin{aligned}
\mathcal{P}_{i \rightarrow f}= & \frac{1}{2 \pi \hbar} \int_{-\infty}^{\infty} d p \\
& \times \int_{-\infty}^{\infty} d q P_{i}^{\mathrm{CTV}}\left(q, p, t_{0}\right) e^{i\left(t-t_{0}\right) \mathcal{L}^{\mathrm{CV}}} P_{f}^{\mathrm{CV}}\left(q, p, t_{0}\right) \\
= & \frac{1}{2 \pi \hbar} \int_{-\infty}^{\infty} d p \int_{-\infty}^{\infty} d q P_{i}^{\mathrm{CTV}}\left(q, p, t_{0}\right) \widetilde{P}_{f}^{\mathrm{CV}}(q, p, t),
\end{aligned}
$$

$P_{i}^{\mathrm{CTV}}$ being the CTV form (an ultradistribution function) of the initial state, and $P_{f}^{\mathrm{CV}}$ the $\mathrm{CV}$ form (a distribution function) of the final state. We observe that there is a kind of "contraction" similar to the one occurring, for instance, in the theory of relativity: we could interchange CV by CTV in Eq. (30) without affecting the result of the integration $[10,11]$.

For the CTV ultradistribution function we have an equation [11] analogous to Eq. (21), whose formal solution is given by

$$
P_{i}^{\mathrm{CTV}}(q, p, t)=\exp \left[-i\left(t-t_{0}\right) \mathcal{L}^{\mathrm{CTV}}\right] P_{i}^{\mathrm{CTV}}\left(q, p, t_{0}\right),
$$

with

$$
\mathcal{L}^{\mathrm{CTV}}=\frac{1}{\hbar} \mathcal{H}^{\mathrm{CTV}}\left[\stackrel{\leftrightarrow}{\Gamma}^{\mathrm{CTV}}-\left(\stackrel{\leftrightarrow}{\Gamma}^{\mathrm{CTV}}\right)^{*}\right]
$$

and

$$
\stackrel{\leftrightarrow}{\Gamma}^{\mathrm{CTV}}=\exp \left(-\hbar \overleftarrow{D}^{*} \vec{D} / 2\right)
$$

We use the model and method described by Lee and Scully $[1,3]$ for the one-dimensional atom-molecule collinear collision, but in the GWP phase-space formulation. Here also in this nonreactive process the atom is treated like a free classical structureless particle while the molecule is represented by a harmonic oscillator. The interaction between the atom and the molecule [13] is the exponential repulsion described in Sec. I. The Hamiltonian for this system in the CV representation is (see Appendix A)

$$
\mathcal{H}^{\mathrm{CV}}=\frac{P^{2}}{2 M}+\frac{p^{2}}{2 m}+\frac{1}{2} k q^{2}+V_{\mathrm{eff}} e^{-\alpha(Q-q)}+\frac{\hbar}{4}\left(\frac{a_{0}^{2}}{m}+\frac{k}{a_{0}^{2}}\right),
$$

where $Q$ and $q$ are the translational and vibrational coordinates respectively, $P$ and $p$ their respective momenta, $V_{\text {eff }}=V_{0} \exp \left(\alpha^{2} \hbar / 4 a_{0}^{2}\right)$ and the parameters $M, m, V_{0}, \alpha$, and $k$ (elastic constant) are taken from Ref. [13], fitting the $\mathrm{He}-\mathrm{H}_{2}$ system. The CTV form of the Hamiltonian is obtained from Eq. (31) by replacing $a_{0}^{2}$ by $-a_{0}^{2}$ [11]. From here on we shall use $m \omega$ for the parameter $a_{0}^{2}$.

In the $\hbar$ power-series expansion of $\mathcal{L}^{\mathrm{CV}}$

$$
\mathcal{L}^{\mathrm{CV}}=\mathcal{L}_{0}+\hbar \mathcal{L}_{1}+\hbar^{2} \mathcal{L}_{2}+\cdots
$$

the contributions of the coordinates $Q$ and $P$ were neglected, except in the zeroth order term given by

$$
\mathcal{L}_{0}=i\left(\frac{\partial \mathcal{H}^{\mathrm{CV}}}{\partial Q} \frac{\partial}{\partial P}-\frac{\partial \mathcal{H}^{\mathrm{CV}}}{\partial P} \frac{\partial}{\partial Q}+\frac{\partial \mathcal{H}^{\mathrm{CV}}}{\partial q} \frac{\partial}{\partial p}-\frac{\partial \mathcal{H}^{\mathrm{CV}}}{\partial p} \frac{\partial}{\partial q}\right)
$$

and which corresponds to classical motion.

In this approximation

$$
D^{n+1} \mathcal{H}^{\mathrm{CV}}=\left(\alpha / a_{0}\right)^{n+1} V_{\text {eff }} \exp [-\alpha(Q-q)] \text { for } n \geqslant 1
$$

So Eq. (29) can then be written 


$$
\begin{aligned}
w\left(t \mid t_{0}\right)= & e^{-i\left(t-t_{0}\right) \mathcal{L}_{0} w_{0}}\left(t_{0} \mid t_{0}\right)+\frac{2 V_{\mathrm{eff}}}{\hbar} \int_{t_{0}}^{t} d t^{\prime} e^{-i\left(t-t^{\prime}\right) \mathcal{L}_{0}} \\
& \times \operatorname{Im}\left[e^{-\alpha(Q-q)} \sum_{n=2}^{\infty} \frac{\left(\frac{\hbar \alpha}{2 a_{0}} D^{*}\right)^{n}}{n !} w\left(t^{\prime} \mid t_{0}\right)\right] .
\end{aligned}
$$

Substituting Eq. (34) into Eq. (28), we have, in first iteration,

$$
\begin{aligned}
P^{\mathrm{CV}}(q, p, t)= & e^{-i\left(t-t_{0}\right) \mathcal{L}_{0}} P^{\mathrm{CV}}\left(q, p, t_{0}\right)+\frac{2 V_{\mathrm{eff}}}{\hbar} \\
& \times \int_{t_{0}}^{t} d t^{\prime}\left[e^{-i\left(t-t^{\prime}\right) \mathcal{L}_{0}} e^{-\alpha(Q-q)}\right] e^{-i\left(t-t^{\prime}\right) \mathcal{L}_{0}} \\
& \times \operatorname{Im} \sum_{n=2}^{\infty} \frac{\left(\frac{\hbar \alpha}{2 a_{0}} D^{*}\right)^{n}}{n !} \\
& \times e^{-i\left(t^{\prime}-t_{0}\right) \mathcal{L}_{0} P^{\mathrm{CV}}}\left(q, p, t_{0}\right) .
\end{aligned}
$$

In order to obtain $\widetilde{P}^{\mathrm{CV}}(q, p, t)=\exp \left[i\left(t-t_{0}\right) \mathcal{L}^{\mathrm{CV}}\right]$ $\times P_{f}^{C V}\left(q, p, t_{0}\right)$, which is the function appearing in Eq. (30), we proceed in the same fashion, but now as the Green's function is given by $\widetilde{w}\left(t \mid t_{0}\right)=\exp \left[i\left(t-t_{0}\right) \mathcal{L}^{\mathrm{CV}}\right] w\left(t_{0} \mid t_{0}\right)$, we get, instead of Eq. (35),

$$
\begin{aligned}
\widetilde{P}^{\mathrm{CV}}(q, p, t)= & e^{i\left(t-t_{0}\right) \mathcal{L}_{0}} P^{\mathrm{CV}}\left(q, p, t_{0}\right)-\frac{2 V_{\mathrm{eff}}}{\hbar} \\
& \times \int_{t_{0}}^{t} d t^{\prime}\left[e^{i\left(t-t^{\prime}\right) \mathcal{L}_{0}} e^{-\alpha(Q-q)}\right] e^{i\left(t-t^{\prime}\right) \mathcal{L}_{0}} \\
& \times \operatorname{Im} \sum_{n=2}^{\infty} \frac{\left(\frac{\hbar \alpha}{2 a_{0}} D^{*}\right)^{n}}{n !} \\
& \times e^{i\left(t^{\prime}-t_{0}\right) \mathcal{L}_{0}} P^{\mathrm{CV}}\left(q, p, t_{0}\right)
\end{aligned}
$$

since $\widetilde{P}^{\mathrm{CV}}\left(q, p, t_{0}\right)=P^{\mathrm{CV}}\left(q, p, t_{0}\right)$.

Substituting Eq. (36) into Eq. (30) we have, with quantum corrections in all orders of $\hbar$,

$$
\begin{aligned}
\mathcal{P}_{i \rightarrow f}= & \int \frac{d p d q}{2 \pi \hbar} P_{i}^{\mathrm{CTV}}\left(q, p, t_{0}\right) e^{i\left(t-t_{0}\right) \mathcal{L}_{0}} P_{f}^{\mathrm{CV}}\left(q, p, t_{0}\right) \\
& -\frac{\alpha V_{\mathrm{eff}}}{a_{0}} \int \frac{d p d q}{2 \pi \hbar} P_{i}^{\mathrm{CTV}}\left(q, p, t_{0}\right) \\
& \times \int_{t_{0}}^{t} d t^{\prime}\left[e^{i\left(t-t^{\prime}\right) \mathcal{L}_{0}} e^{-\alpha(Q-q)}\right] \cdot e^{i\left(t-t^{\prime}\right) \mathcal{L}_{0}} \\
& \times \operatorname{Im}\left[\left(\int_{0}^{1} d \xi e^{\left(\xi \hbar \alpha / 2 a_{0}\right) D^{*}}-1\right) D^{*} e^{i\left(t^{\prime}-t_{0}\right) \mathcal{L}_{0}}\right. \\
& \left.\times P_{f}^{\mathrm{CV}}\left(q, p, t_{0}\right)\right],
\end{aligned}
$$

where we have used the property, valid for any operator $A$,

$$
\int_{0}^{1} d \xi e^{\xi A}=\sum_{n=0}^{\infty} \frac{A^{n}}{(n+1) !} .
$$

The first term in Eq. (37) is the CA which is the zerothorder term of our $\hbar$ power-series expansion, and corresponds to the expression of the SQC method of Lee and Scully [1]. The difference between these two expressions lies in the distribution functions; while Lee and Scully worked with the product of two Wigner distribution functions, in Eq. (37) we have the product of two Gaussian distribution functions, one of them in the CTV form and the other in the CV form.

Like in the SQC method, $[1,3]$ here $P_{i}^{\mathrm{CTV}}\left(q, p, t_{0}\right)$ represents the initial vibrational state of the system, which we propagate along classical trajectories. We also use the Lee and Scully method [1] for constructing the two-dimensional rectangular grid in the $(q, p)$ plane. $P_{i}^{\mathrm{CTV}}\left(q_{n}, p_{n}, t_{0}=-\infty\right)$ is the weight carried by the point $\left(q_{n}, p_{n}\right)$ of the $n$th cell of the grid.

After integrating Hamilton's equations for each point of the grid, the final GDF $\left[P_{f}^{\mathrm{CV}}(q, p, t=\infty)\right]$ is calculated, and then the transition probabilities are computed in the CA which corresponds to the first term in Eq. (37).

The GDF in the CV form is given by (see Appendix B)

$$
P_{n}^{\mathrm{CV}}(y)=\frac{1}{n !} y^{n} e^{-y}
$$

TABLE I. Transition probability $\mathcal{P}_{0 \rightarrow f}$ for a collinear $\mathrm{He}-\mathrm{H}_{2}$ collision calculated by the quantum-mechanical (QM) method (Ref. [13]), statistical quasiclassical (SQC) method (Ref. [1]), and Gaussian wave-packet (GWP) method in the CA [first term in Eq. (37)]. The total initial energy $E$ is measured in units of $\hbar \omega / 2$, where $\omega$ is the vibrational frequency of the $\mathrm{H}_{2}$ molecule. In the calculation of $P_{0}^{\mathrm{CTV}}$ at least 100 Laguerre polynomials were used, although only about ten polynomials are required in order to obtain convergence. Numbers inside brackets give the upper limit of the transition probability, and $*$ means that the transition is prohibited classically.

\begin{tabular}{ccccc}
\hline \hline$E$ & $\mathcal{P}_{0 \rightarrow f}$ & QM & SQC & GWP \\
\hline 8 & $0 \rightarrow 0$ & $(0.892)$ & 0.893 & 0.830 \\
& $0 \rightarrow 1$ & 0.108 & 0.107 & 0.156 \\
& $0 \rightarrow 2$ & 0.001 & & 0.014 \\
12 & $0 \rightarrow 0$ & $(0.538)$ & 0.529 & 0.501 \\
& $0 \rightarrow 1$ & 0.394 & 0.412 & 0.349 \\
& $0 \rightarrow 2$ & 0.068 & 0.068 & 0.122 \\
& $0 \rightarrow 3$ & & & 0.028 \\
16 & $0 \rightarrow 0$ & $(0.204)$ & 0.187 & 0.229 \\
& $0 \rightarrow 1$ & 0.434 & 0.422 & 0.339 \\
& $0 \rightarrow 2$ & 0.291 & 0.314 & 0.250 \\
& $0 \rightarrow 3$ & 0.071 & 0.077 & 0.124 \\
& $0 \rightarrow 4$ & & & 0.045 \\
& $0 \rightarrow 5$ & & & 0.013 \\
& $0 \rightarrow 0$ & $(0.060)^{*}$ & $0.046^{*}$ & $0.090^{*}$ \\
& $0 \rightarrow 1$ & 0.218 & 0.202 & 0.221 \\
& $0 \rightarrow 2$ & 0.366 & 0.351 & 0.270 \\
& $0 \rightarrow 3$ & 0.267 & 0.294 & 0.220 \\
& $0 \rightarrow 4$ & 0.089 & 0.106 & 0.134 \\
& $0 \rightarrow 5$ & & & 0.066 \\
\hline \hline & & & &
\end{tabular}


TABLE II. Similar to Table I, except that the oscillator goes from the first exited state to the final state $f$.

\begin{tabular}{ccccc}
\hline \hline$E$ & $\mathcal{P}_{1 \rightarrow f}$ & QM & SQC & GWP \\
\hline 8 & $1 \rightarrow 0$ & 0.108 & 0.106 & 0.135 \\
& $1 \rightarrow 1$ & $(0.850)$ & 0.863 & 0.780 \\
& $1 \rightarrow 2$ & 0.042 & 0.031 & 0.085 \\
12 & $1 \rightarrow 0$ & 0.394 & 0.411 & 0.396 \\
& $1 \rightarrow 1$ & $(0.244)$ & 0.176 & 0.250 \\
& $1 \rightarrow 2$ & 0.345 & 0.385 & 0.272 \\
& $1 \rightarrow 3$ & $0.037^{*}$ & $0.028^{*}$ & $0.082^{*}$ \\
16 & $1 \rightarrow 0$ & 0.434 & 0.420 & 0.377 \\
& $1 \rightarrow 1$ & $(0.034)$ & 0.065 & 0.137 \\
& $1 \rightarrow 2$ & 0.220 & 0.151 & 0.176 \\
& $1 \rightarrow 3$ & 0.261 & 0.302 & 0.181 \\
& $1 \rightarrow 4$ & 0.051 & 0.061 & 0.098 \\
& $1 \rightarrow 5$ & & & 0.031 \\
20 & $1 \rightarrow 0$ & 0.218 & 0.199 & 0.230 \\
& $1 \rightarrow 1$ & $(0.286)$ & 0.285 & 0.223 \\
& $1 \rightarrow 2$ & 0.009 & 0.042 & 0.143 \\
& $1 \rightarrow 3$ & 0.170 & 0.090 & 0.149 \\
& $1 \rightarrow 4$ & 0.240 & 0.262 & 0.149 \\
& $1 \rightarrow 5$ & 0.077 & 0.121 & 0.105 \\
\hline \hline
\end{tabular}

where $y=E / \hbar \omega, E(q, p)$ being the energy of the classical harmonic oscillator, and $n$ is the quantum number which corresponds to the $n$th eigenstate of the harmonic oscillator. Introducing the new variable $r^{2}=y$, the GDF in the CTV form is (see Appendix B)

$$
P_{n}^{\mathrm{CTV}}(r)=e^{-(1 / 4 r)(\partial / \partial r) r(\partial / \partial r)} P_{n}^{\mathrm{CV}}(r) .
$$

\section{NUMERICAL RESULTS AND CONCLUSIONS}

Using the mapped form of the quantum Liouville equation in GWP phase space, we have derived expressions for the transition probabilities for semiclassical calculations of inelastic atom-molecule collisions which include also the quantum terms. These expressions are similar to those obtained in Ref. [9] for the WW formalism.

We show in Tables I and II the transition probabilities $\mathcal{P}_{i \rightarrow f}$ from the initial $(i)$ to the final $(f)$ state of the $\mathrm{He}-\mathrm{H}_{2}$ system which were computed, first, by integrating numerically the Schrödinger equation (these are the exact quantum mechanical results taken from Ref. [13]), second, by using the Weyl-Wigner representation in the statistical quasiclassical method taken from Ref. [1], and, third, by using the Gaussian wave-packet representation in the causal approximation. The last ones are our results, and they are given by the first term in Eq. (37). For more details on the system and models, see the mentioned references.

The results in Tables I and II, obtained using only the zeroth-order term for both methods, SQC and GWP, should improve if quantum corrections are introduced. The accuracy of the SQC and the GWP methods was checked by studying how well the transition probabilities obey microscopic reversibility, the results are presented in Table III. These results confirm that, for the CA, the amount of error coming
TABLE III. Similar to Table I; results of Tables I and II are compared in order to test microscopic reversibility.

\begin{tabular}{ccccc}
\hline \hline$E$ & $\mathcal{P}_{i \rightarrow f}$ & QM & SQC & GWP \\
\hline \multirow{2}{*}{8} & $0 \rightarrow 1$ & 0.108 & 0.107 & 0.156 \\
& $1 \rightarrow 0$ & 0.108 & 0.106 & 0.135 \\
12 & $0 \rightarrow 1$ & 0.394 & 0.412 & 0.349 \\
& $1 \rightarrow 0$ & 0.394 & 0.411 & 0.396 \\
16 & $0 \rightarrow 1$ & 0.434 & 0.422 & 0.339 \\
& $1 \rightarrow 0$ & 0.434 & 0.420 & 0.377 \\
20 & $0 \rightarrow 1$ & 0.218 & 0.202 & 0.221 \\
& $1 \rightarrow 0$ & 0.218 & 0.199 & 0.230 \\
\hline \hline
\end{tabular}

from propagating the WDF along classical trajectories is less than that coming from propagating the GWP distribution along the classical trajectories, as stated in Ref. [3]. This agrees with the vanishing result found in Sec. II for the first quantum correction to the $\mathrm{CA}$ in the $\mathrm{WW}$ formalism. However, preliminary calculations of the first quantum correction in the GWP formalism gave unsatisfactory nonzero results. We attribute this failure to the fact that in the GWP approach the calculation of transition probabilities, Eq. (37), involves two GDF's, one in a CTV form (which is singular), and the other in a CV form (which is smooth). For the numerical procedure we approximated the Dirac $\delta$ functions and derivatives, present in the CTV form, by finite sums over the Laguerre polynomials, as described in Appendix B. However, by increasing the number of terms in the sums, we were not able to obtain stable results for the quantum corrections, thus no inference could be extracted for their estimation.

We also remark here that, since the contravariant GWP distributions involve $\delta$ functions and its derivatives calculated at the origin of the phase space (see Appendix B) in the transition probability given by Eq. (37), only those trajectories ending with the oscillator at rest at the origin will contribute, whereas on the WW picture [Eq. (11)], at initial and final times, the contributing trajectories correspond to the oscillator having a range of energies of the order of $\hbar \omega$. This difference has to be compensated for by larger contributions of the quantum corrections, and, thus, possibly many terms in the expansion of Eq. (32) are required to yield results of similar accuracy.

In conclusion, to calculate transition probabilities semiclassically, the WW formalism seems more advantageous than the GWP one, since the former involves two Wigner functions (initial and final states) which, although not as smooth (and positive) as the CV form of the GDF's, possess the virtue of not having strong singularities as those occuring in the CTV form. Finally, we mention that in the CA, both methods (SQC and GWP) give nonvanishing results for classically forbidden processes, and the numerical calculations for both formalisms present almost the same degree of difficulty for being performed.

\section{ACKNOWLEDGMENTS}

M.C.T. would like to thank the Instituto de Física Teórica for hospitality. S.S.M. acknowledges partial financial support 
from the Conselho Nacional de Desenvolvimento Científico e Tecnológico (CNPq), Brazil, and CNPq-FAPESP cooperative Grant No. 400285/95-9.

\section{APPENDIX A: HAMILTONIAN IN THE CV REPRESENTATION FOR THE ATOM-MOLECULE SYSTEM}

Let $|p q\rangle$ be the minimum uncertainty $\operatorname{GWP}$ and $(p, q)$ a point in the phase space, $\hat{p}$ and $\hat{q}$ the linear momentum and position operators, respectively, and $F(\hat{p}, \hat{q})$ an arbitrary function expandable in a power series of its arguments. The ordering of the operators in each term of the series is specified by the function $F$. In Refs. [10-12] it was shown that the mapped $\mathrm{CV}$ form of $F(\hat{p}, \hat{q})$ can be obtained by using the algorithm

$f_{F}(p, q) \equiv F\left(p-\frac{i \hbar}{2} \frac{\partial}{\partial q}+\frac{\hbar a_{0}^{2}}{2} \frac{\partial}{\partial p}, q+\frac{i \hbar}{2} \frac{\partial}{\partial p}+\frac{\hbar}{2 a_{0}^{2}} \frac{\partial}{\partial q}\right) 1$,

where the functional form of $F$ is kept unchanged, but the operators $\hat{p}$ and $\hat{q}$ are substituted (keeping the original order) by a differential realization, and, thereafter, the whole expression operates on the unity. For instance, the CV form of $\hat{q}^{2}$ in the GWP representation is

$$
\left\langle p q\left|\hat{q}^{2}\right| p q\right\rangle=q^{2}+\frac{\hbar}{2 a_{0}^{2}}
$$

and that of $\hat{p}^{2}$ is

$$
\left\langle p q\left|\hat{p}^{2}\right| p q\right\rangle=p^{2}+\frac{\hbar a_{0}^{2}}{2} .
$$

The Hamiltonian for the atom-molecule system considered in this work is

$$
H(Q, P, \hat{q}, \hat{p})=\frac{P^{2}}{2 M}+\frac{\hat{p}^{2}}{2 m}+\frac{k \hat{q}^{2}}{2}+V_{0} e^{-\alpha(Q-\hat{q})},
$$

where $Q$ and $P$ are the coordinate and momentum of the atom which is treated like a free classical structureless particle, while the molecule is represented by the quantum harmonic oscillator.

Using the algorithm (A1), we obtain

$$
\left\langle p q\left|e^{-\alpha(Q-\hat{q})}\right| p q\right\rangle=e^{-\alpha(Q-q)+\left(\alpha^{2} \hbar / 4 a_{0}^{2}\right)} .
$$

Thus, the CV form of the Hamiltonian (A4) is

$$
\begin{aligned}
\langle p q|H| p q\rangle= & \frac{P^{2}}{2 M}+\frac{p^{2}}{2 m}+\frac{k q^{2}}{2}+V_{\mathrm{eff}} e^{-\alpha(Q-q)} \\
& +\frac{\hbar}{4}\left(\frac{a_{0}^{2}}{m}+\frac{k}{a_{0}^{2}}\right),
\end{aligned}
$$

where $V_{\text {eff }}=V_{0} e^{\alpha^{2} \hbar / 4 a_{0}^{2}}$ is the effective strength of the interaction.

\section{APPENDIX B: DERIVATION OF THE CTV FORM OF THE GDF}

Let $\left|\varphi_{n}\right\rangle$, with $n=0,1,2, \ldots$ be the eigenstate of the harmonic oscillator and $|\alpha\rangle=|p q\rangle$ the GWP representation; the $\mathrm{CV}$ form for the density operator $\left\langle p q \mid \varphi_{n}\right\rangle\left\langle\varphi_{n} \mid p q\right\rangle$ is given by $P_{n}^{\mathrm{CV}}(r)=\left(r^{2 n} / n !\right) e^{-r^{2}}$, where the parameter $\alpha=\left(a_{0}^{2} / 2 \hbar\right)^{1 / 2} q+i\left(1 / 2 \hbar a_{0}^{2}\right)^{1 / 2} p=r e^{i \theta}$, [10,15]. If $E$ represents the energy of the classical harmonic oscillator and $r^{2}=(E / \hbar \omega)=y$, we may write

$$
P_{n}^{\mathrm{CV}}(y)=\frac{1}{n !} y^{n} e^{-y}
$$

thus the CV form of the GDF depends only on the energy of the harmonic oscillator.

In order to obtain the CTV form of the GDF, we use the property of the coherent states [15] which relates the normal (NO) and antinormal (AO) ordering of a function $f$ of $\alpha$ and $\alpha^{*}$

$$
f^{(\mathrm{AO})}\left(\alpha, \alpha^{*}\right)=e^{\left(\partial^{2} / \partial \alpha \partial \alpha^{*}\right)} f^{(\mathrm{NO})}\left(\alpha, \alpha^{*}\right) .
$$

In polar coordinates $r$ and $\theta$, we have

$$
\frac{\partial^{2}}{\partial \alpha \partial \alpha^{*}}=\frac{1}{4 r}\left(\frac{\partial}{\partial r} r \frac{\partial}{\partial r}+\frac{1}{r} \frac{\partial^{2}}{\partial \theta^{2}}\right),
$$

and Eq. (B2) in the GWP representation is written [10]

$$
P_{n}^{\mathrm{CTV}}(r, \theta)=e^{-(1 / 4 r)\left[(\partial / \partial r) r(\partial / \partial r)+(1 / r)\left(\partial^{2} / \partial \theta^{2}\right)\right]} P_{n}^{\mathrm{CV}}(r, \theta) .
$$

For any positive integer $n$, we derived the formula

$$
\left(\frac{1}{4 r} \frac{\partial}{\partial r} r \frac{\partial}{\partial r}\right)^{n} e^{-r^{2}}=\left(\frac{d}{d y} y \frac{d}{d y}\right)^{n} e^{-y}=(-1)^{n} n ! L_{n}(y) e^{-y},
$$

where $L_{n}$ are the Laguerre polynomials. Since $P_{n}^{\mathrm{CV}}(r)$ for the harmonic oscillator does not depend on $\theta$, Eq. (B4) can be written as

$$
P_{n}^{\mathrm{CTV}}(y)=e^{-(d / d y) y(d / d y)} P_{n}^{\mathrm{CV}}(y) .
$$

Now, we call attention to the fact that the CTV distribution functions are singular; actually, they are ultradistributions. They will depend on the "moments" $\sum_{n=0}^{\infty} n^{s} L_{n}(y)$, where the Laguerre polynomials act as "weight" functions and $s$ is an integer. These "moments" are written in terms of the Dirac $\delta$ function and its derivatives. For instance, for $s=0,1,2$ we have

$$
\begin{gathered}
\sum_{n=0}^{\infty} L_{n}(y)=\delta(y), \\
\sum_{n=0}^{\infty} n L_{n}(y)=\delta^{\prime}(y)-\delta(y), \\
\sum_{n=0}^{\infty} n^{2} L_{n}(y)=2 \delta^{\prime \prime}(y)-3 \delta^{\prime}(y)+\delta(y) .
\end{gathered}
$$


Using Eqs. (B6), Eq. (B1), and Eq. (B5), we obtain, for $n=0$,

$$
P_{0}^{\mathrm{CTV}}(y)=\sum_{n=0}^{\infty} L_{n}(y) e^{-y}=e^{-y} \delta(y)
$$

For $n>0$ we introduce the auxiliary function

$$
P_{0}^{\mathrm{CTV}}(y, \beta)=e^{-(d / d y) y(d / d y)} e^{-\beta y}=\sum_{n=0}^{\infty} \beta^{n} L_{n}(\beta y) e^{-\beta y}
$$

in order to write

$$
P_{n}^{\mathrm{CTV}}(y)=\frac{1}{n !} \lim _{\beta \rightarrow 1}\left(-\frac{\partial}{\partial \beta}\right)^{n} P_{0}^{\mathrm{CTV}}(y, \beta) .
$$

For $n=1,2$, we obtain, respectively,

$$
P_{1}^{\mathrm{CTV}}(y)=\sum_{n=0}^{\infty}(1-n) L_{n}(y) e^{-y}=e^{-y}\left[-\delta^{\prime}(y)+2 \delta(y)\right]
$$

and

$$
\begin{aligned}
P_{2}^{\mathrm{CTV}}(y) & =\sum_{n=0}^{\infty}\left(n^{2}-5 n+2\right) L_{n}(y) e^{-y} \\
& =e^{-y}\left[\delta^{\prime \prime}(y)-4 \delta^{\prime}(y)+4 \delta(y)\right]
\end{aligned}
$$

where we used Eqs. (B7)-(B9).
[1] H. W. Lee and M. O. Scully, J. Chem. Phys. 73, 2238 (1980). [2] M. Hillery, R. F. O'Connel, M. O. Scully, and E. P. Wigner, Phys. Rep. 106, 121 (1984).

[3] H. W. Lee, Phys. Rep. 259, 147 (1995), and references therein. [4] G. W. Bund, J. Phys. A A28, 3709 (1995).

[5] P. Carruthers and F. Zachariasen, Rev. Mod. Phys. 55, 245 (1983).

[6] H. Esbensen, in Nuclear Structure and Heavy Ion Collisions, Proceedings of the International School of Physics "Enrico Fermi,', Course LXXVII, Varenna Lake Como, 1979, edited by R. A. Broglia, R. A. Ricci and C. H. Dasso (North-Holland, Amsterdam, 1981).
[7] D. L. Bunker, Math. Comput. Phys. 10, 287 (1971).

[8] E. J. Heller, J. Chem. Phys. 65, 1289 (1976).

[9] G. W. Bund, S. S. Mizrahi, and M. C. Tijero, Phys. Rev. A 53, 1191 (1996).

[10] S. S. Mizrahi, Physica A 127, 241 (1984).

[11] S. S. Mizrahi, Physica A 135, 237 (1986).

[12] M. C. Tijero, Ph.D. thesis, IFT-T.004/94, 1994 (unpublished).

[13] D. Secrest and B. R. Johnson, J. Chem. Phys. 45, 4556 (1966).

[14] S. R. de Groot and L. G. Suttorp, Foundations of Electrodynamics (North-Holland, Amsterdam, 1972), Chap. VI.

[15] W. H. Louisell, in Quantum Statistical Properties of Radiation (Wiley, New York, 1973). 